\title{
IMMUNOSTAINING OF NEUROFIBRILLARY TANGLES IN ALZHEIMER'S SENILE DEMENTIA WITH A NEUROFILAMENT ANTISERUM $^{1}$
}

\author{
DORIS DAHL, ${ }^{*, 2}$ DENNIS J. SELKOE, $\ddagger$ ROBERT T. PERO, ${ }^{*}$ AND AMICO BIGNAMI* \\ ${ }^{*}$ Spinal Cord Injury Research, West Roxbury Veterans Administration Medical Center, and $\ddagger$ Mailman Research Center, \\ McLean Hospital, Department of Neuropathology, Harvard Medical School, Boston, Massachusetts 02115
}

Received June 2, 1981; Revised September 24, 1981; Accepted Scptember 24, 1981

\begin{abstract}
Using anti-chicken brain neurofilament antisera, Alzheimer's neurofibrillary tangles from two patients with senile dementia were stained by immunofluorescence and by the peroxidase-antiperoxidase procedure in cryostat sections of hippocampus and frontal cortex. In sections of cerebellum obtained from the same patients, the distribution of immunostaining was the same as that observed in experimental animals: Purkinje cell baskets and nerve fibers in the inner half of the molecular layer were demonstrated selectively. The immunostaining of the tangles was abolished when the antisera were absorbed by their own antigen, by bovine brain filament preparations, or by the fraction of bovine brain filament preparations nonabsorbed on anti-glial fibrillary acidic (GFA) protein immunoaffinity columns. Absorption with a bovine microtubule preparation isolated by two cycles of the assembly-disassembly procedure did not abolish the staining. Immunostaining experiments conducted on bovine brain filament preparations resolved on sodium dodecyl sulfate-polyacrylamide gel electrophoresis showed that the antisera staining the tangles reacted with the $200,000-, 150,000$-, and 70,000-dalton neurofilament polypeptides. Antisera raised to the 150,000 dalton bovine neurofilament polypeptide isolated by sodium dodecyl sulfate-polyacrylamide gel electrophoresis stained the tangles much less intensely, although Purkinje cell baskets in the cerebellum appeared well stained. No staining of neurofibrillary tangles was observed with antisera to other classes of 10-nm filament proteins (GFA protein, vimentin, and desmin).
\end{abstract}

The neurofibrillary tangle is a structural alteration affecting neurons during normal and pathological aging of the human brain. The lesions consist of masses of abnormal fibrils found within the cytoplasm of certain neuronal perikarya and identified at the light microscopic level with silver stains. As far as is known, neurofibrillary tangles are unique to the human brain, since they have not been identified in aged non-human primates and lower mammals (Wiśniewski et al., 1973). Small numbers of neurofibrillary tangles are found within neurons of

\footnotetext{
${ }^{1}$ This work was supported by National Science Foundation Grant BNS-7912962 (D. D.), National Institutes of Health Grant AG-01307 (D. J. S.), and by the Veterans Administration (A. B. and D. D.). Dr. Selkoe is the recipient of a Teacher-Investigator Award from the National Institute of Neurological and Communicative Disorders and Stroke. Mr. Pero was supported by a Veterans Administration Summer Student Stipend. We thank Dr. Klaus Weber for the gift of vimentin antisera and Dr. Friederich H. Klutzow for brain samples of Alzheimer's dementia.

${ }^{2}$ To whom correspondence should be addressed at Spinal Cord Injury Research, Veterans Administration Medical Center, 1400 VFW Parkway, West Roxbury, MA 02132.
}

hippocampus and amygdala in a majority of neurologically normal individuals after age 70 . The number of tangle-bearing hippocampal neurons is 6 to 40 times greater in patients with dementia of the Alzheimer type (Ball, 1976), which is the most common cause of progressive intellectual failure in late life (Katzman, 1976). In Alzheimer's disease, neurofibrillary tangles also occur with high frequency in many areas of cerebral cortex besides hippocampus. Their density appears to correlate strongly with the duration and severity of the dementia (Tomlinson et al., 1970; Farmer et al., 1976).

The distinctive electron microscopic appearance of the neurofibrillary tangle was first described by Terry (1963) and Kidd (1963). It is formed by bundles of filaments approximately $20 \mathrm{~nm}$ in diameter and characterized by constrictions about $10 \mathrm{~nm}$ in diameter at $80-\mathrm{nm}$ intervals. These have been interpreted as paired filaments coiled as double helixes making a full turn every $160 \mathrm{~nm}$ (Kidd, 1963; Wiśniewski et al., 1976) or, alternatively, as constricted microtubules, for which the term twisted tubules was proposed (Terry, 1963).

The chemical composition of the paired helical fila- 
ments in aged and diseased neurons is still controversial (Grundke-Iqbal et al., 1979a; Eng et al., 1980). The topographic selectivity of neurofibrillary tangles in the cerebral cortex, with sparing of many neuronal and nonneuronal elements, makes their quantitative purification problematic. For this reason, an antiserum which can react reliably with Alzheimer's neurofibrillary tangles would provide an important tool for further morphological and biochemical investigations.

\section{Materials and Methods}

Antisera to neurofilament proteins isolated by hydroxyapatite chromatography from $8 \mathrm{M}$ urea extracts of chicken brain were raised in three New Zealand albino rabbits as described (Dahl and Bignami, 1977; Dahl, 1980a). The antisera were shown to react with the $\sim 150,000$ - and 72,000-dalton polypeptides of the mammalian neurofilament "triplet" (Hoffman and Lasek, 1975; Schlaepfer and Freeman, 1978; Thorpe et al., 1979; Lazarides, 1980) by immunoaffinity chromatography (Dahl, 1981). They also were shown to stain neurofibrils selectively by indirect immunofluorescence and by the peroxidase-antiperoxidase (PAP) procedure in cryostat sections (Dahl and Bignami, 1977; Dahl et al., 1981a) and to decorate neurofilaments at the electron microscopic level (Dahl et al., 1981b). Absorption experiments were conducted as previously reported (Dahl and Bignami, 1979). The antisera were absorbed by their own antigen, $100 \mu \mathrm{g}$ of protein $/ \mathrm{ml}$, bovine brain filament preparations, $300 \mu \mathrm{g}$ of protein $/ \mathrm{ml}$, the fraction of a bovine brain filament preparation not absorbed on anti-glial fibrillary acidic (GFA) immuno-Sepharose, $250 \mu \mathrm{g}$ of protein $/ \mathrm{ml}$ (Dahl and Bignami, 1979), and by a microtubule preparation isolated from bovine brain by two cycles of the assembly-disassembly procedure, $1 \mathrm{mg}$ of protein $/ \mathrm{ml}$. Antisera to the $\sim 150,000$-dalton neurofilament polypeptide isolated by sodium dodecyl sulfate-polyacrylamide gel electrophoresis (SDS-PAGE) from bovine brain filament preparations were prepared as reported (Dahl, $1980 \mathrm{~b})$. Antisera to GFA protein, vimentin, and desmin were prepared as previously described (Dahl and Bignami, 1976, 1981; Franke et al., 1978). Indirect immunofluorescence with rhodamine- and fluorescein-conjugated antisera and immunostaining with the PAP procedure were conducted on cryostat sections. Immunoreagents were from Cappel Laboratories, Cochranville, PA. The dilutions of the primary antisera varied from 1:5 to 1:40 for immunofluorescence and from 1:50 to 1:500 for the PAP procedure. The low dilutions were used to confirm negative results (i.e., lack of immunostaining). Blocks of frontal cortex, hippocampus, and cerebellum were dissected 18 and $4 \mathrm{hr}$ postmortem, respectively, from a 67 year-old woman and a 59-year-old man who had suffered from progressive severe dementia for 5 to 10 years prior to death. The clinical diagnosis of Alzheimer's disease was confirmed by routine histological examination. Immunostaining of gels was conducted on bovine brain filament preparations (De Vries et al., 1972) resolved on SDS-PAGE (Weber and Osborn, 1969). For immunofluorescence labeling (Gröschel-Stewart et al., 1976), the gels loaded with approximately $100 \mu \mathrm{g}$ of brain filament protein were incubated with 1:5 and 1:10 dilutions of the primary antisera. Immunoperoxidase staining by the PAP procedure was conducted according to Van Raamsdonk et al. (1977). Longitudinal cryostat sections $100 \mu \mathrm{m}$ thick were fixed for $10 \mathrm{~min}$ in $1 \%$ formalin at $4^{\circ} \mathrm{C}$. The sections were washed in phosphate-buffered saline, $\mathrm{pH}$ 7.5 , for $10 \mathrm{~min}$ and incubated overnight at $4^{\circ} \mathrm{C}$ in $1: 50$ dilutions of the primary antisera. A $30-\mathrm{min}$ wash in phosphate buffer at room temperature and a distilled water rinse were followed by incubation with goat antirabbil IgG at 1:10 dilutions for $60 \mathrm{~min}$ at room temperature. The phosphate buffer wash was repeated and the sections were incubated with PAP at 1:25 dilutions for 60 min at room temperature. The sections then were rinsed with phosphate buffer for $30 \mathrm{~min}$ at room temperature and dipped for $1 \mathrm{~min}$ in a $0.05 \mathrm{~m}$ Tris solution, $\mathrm{pH}$ 7.6. While the sections were still moist, the substrate diaminobenzidine activated with $\mathrm{H}_{2} \mathrm{O}_{2}$ was added. After a few minutes, the brown stain appeared. Finally, the gel sections were rinsed in distilled water and mounted in glycerol. Preimmune sera were used as a control in each experiment.

\section{Results}

Due to the large amount of autofluorescent lipofuscin pigment in aged human brain, most observations were made with rhodamine-conjugated antisera. We found that, in freshly cut sections of human cortex, lipofuscin pigment was more brightly autofluorescent with fluorescein optics than with rhodamine optics. By changing filters in the fluorescence microscope, the distinction between immunofluorescence and autofluorescence of intraneuronal material thus was easily made.

The specificity of the neurofilament antisera in autopsied human brain was assessed in the cerebellum. As originally reported in the rat (Dahl and Bignami, 1977), the following criteria allowed the characterization of our neurofilament antisera: (i) intense staining of Purkinje cell baskets and of a delicate mesh of nerve fibers in the lower half of the molecular layer and (ii) absence of staining of Purkinje cells (perikarya and dendrites) and of Bergmann glia in the molecular layer. As shown in Figures $1 A, 2$, and 5 , these criteria were met when applied to postmortem human brain.

Due to their intense staining, Alzheimer's neurofibrillary tangles were readily identified in the pyramidal layer of the hippocampus with the anti-chicken brain neurofilament antisera using both immunofluorescence and the PAP procedure (Figs. $1, B$ and $C$ and 3). Staining of the tangles with this antiserum was still present following its absorption with a twice-cycled microtubule preparation but disappeared after absorption of the antiserum with its own antigen, with bovine brain filament preparations, or with the fraction of bovine brain filaments not absorbed on an anti-GFA immuno-Sepharose column.

Compared to hippocampus, neurofibrillary tangles in the frontal cortex were fewer in number. The apical dendrites of normal pyramidal neurons were stained. Perinuclear location of immunofluorescence appeared to be a characteristic of the tangle-bearing neurons in both hippocampus and frontal cortex.

Our findings are less conclusive as to the reactivity of the senile plaque with anti-chicken brain neurofilament 

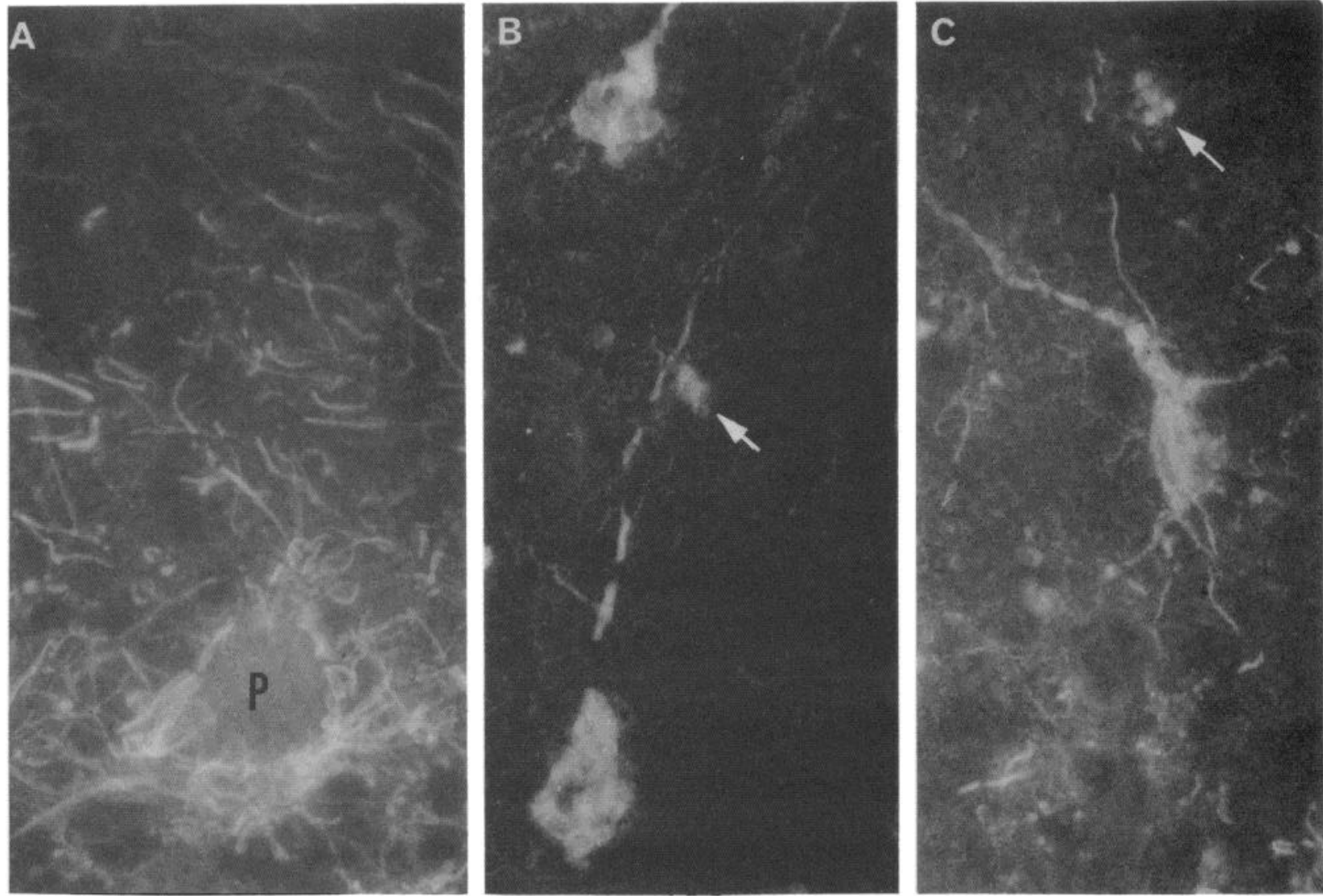

Figure 1. Immunofluorescence with anti-chicken brain neurofilament antisera at 1:40 dilution. $A$, Purkinje cell baskets and nerve fibers in the inner half of the molecular layer, $P$, Purkinje cell body. $B$ and $C$, Alzheimer's neurofibrillary tangles in the pyramidal layer of the hippocampus. Arrows point to autofluorescent pigment (same case as in $A$ ). Magnification $\times 320$.

antisera. Senile plaques are the other characteristic neuropathological finding of Alzheimer's disease. They are formed by a central core of amyloid surrounded by small nerve processes undergoing degeneration (Wiśniewski and Terry, 1973). As illustrated in Figure 4, we observed, in the neuropil of both hippocampus and frontal cortex, small deposits of reaction products often surrounding a central core. We tentatively interpret these findings as indicating that the small nerve processes of the senile plaque react with this neurofilament antiserum, while the central amyloid core does not.

Antisera to the gel-purified 150,000-dalton protein from bovine brain filament preparations appeared to show faint staining of the Alzheimer's tangles, although these antisera stained Purkinje cell baskets in a fashion identical to the anti-chicken brain neurofilament antisera (Fig. 5). Antisera to GFA protein, vimentin, and desmin failed to decorate the tangles.

Immunolabeling of bovine brain filament preparations resolved on SDS-PAGE confirmed previous findings (Gambetti et al., 1980) concerning the reactivity of the anti-chicken antisera with the polypeptides of the neurofilament "triplet." The antisera stained the 200,000 , 150,000 , and 70,000 bands both by immunofluorescence and by the PAP procedure. With the latter method but not by immunofluorescence, weak staining in the 50,000 range was observed also. Antisera to the gel-purified 150,000 neurofilament polypeptide stained the 150,000 band and a band in the 200,000 range, a not unexpected finding since cross-reactivity between these two neurofilament proteins was demonstrated previously (Dahl, $1980 \mathrm{~b})$. No staining was observed in the 70,000 and 50,000 range. GFA antisera, used as a positive control, selectively labeled GFA protein, a major component of bovine brain filament preparations (Dahl and Bignami, 1979). No staining was observed with preimmune sera.

\section{Discussion}

Immunostaining of Alzheimer's neurofibrillary tangles has been reported previously (Grundke-Iqbal et al., 1979a, b; Ishii et al., 1979; Gambetti et al., 1980). Grundke-Iqbal et al. (1979a) carried out immunocytochemical studies of Alzheimer cortex using an antiserum raised against a whole microtubule fraction prepared from normal human brain by two cycles of the assemblydisassembly procedure. This antiserum stained neurofibrillary tangles in paraffin-embedded sections from autopsied Alzheimer brain using the PAP method. Weak or no staining of normal neuronal perikarya was observed. Pre-absorption of the antiserum with its antigen abolished the PAP staining of the neurofibrillary tangles. The antigen(s) within the microtubule preparation that gives rise to the antibodies that stain the tangles has not yet been determined precisely. Iqbal et al. (1980) have reported preliminary results indicating that the immunogenic protein is not tubulin itself nor the neurofilament 


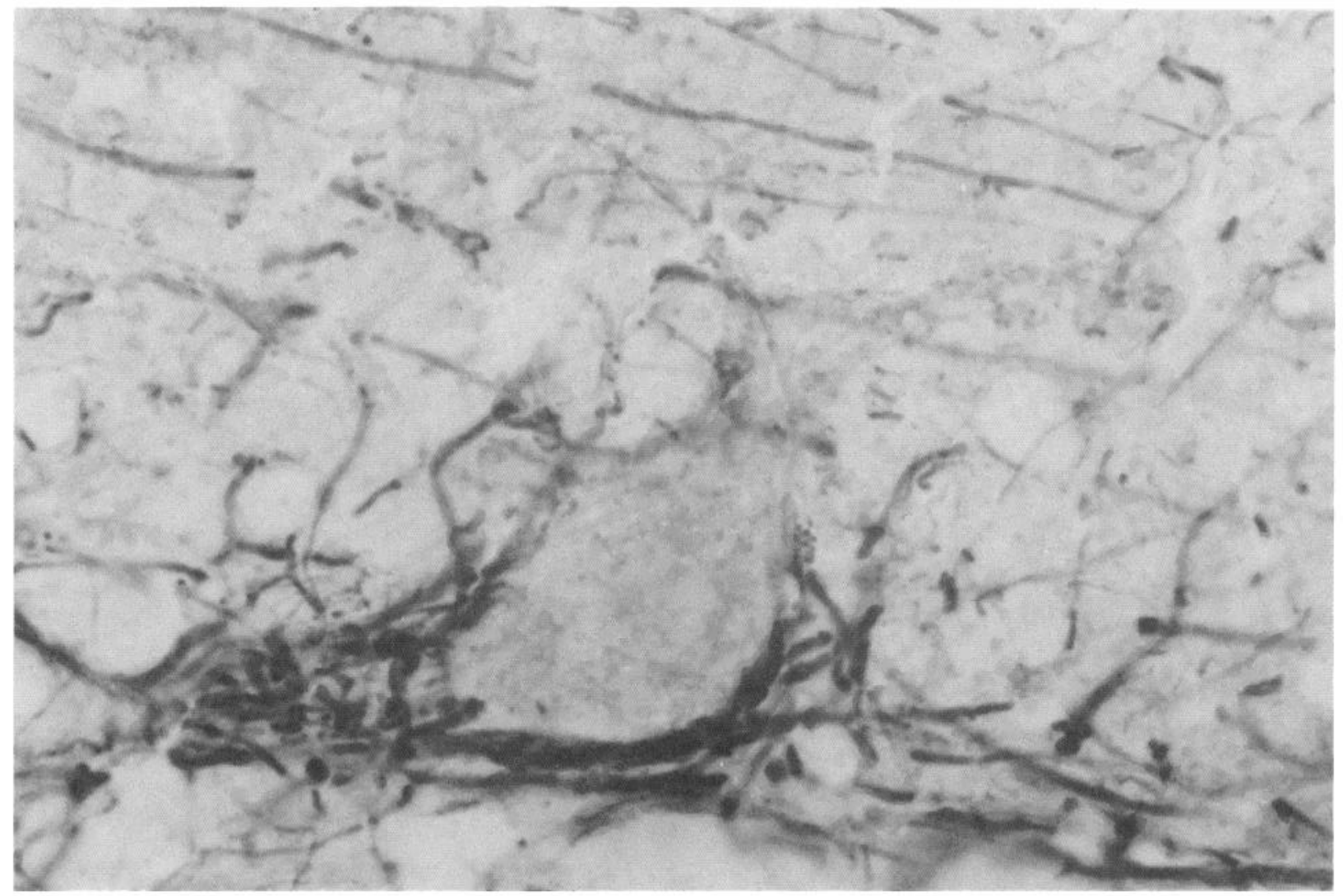

Figure 2. Staining of Purkinje cell baskets and of nerve fibers in the inner part of the molecular layer of human cerebellum using the PAP procedure with anti-chicken brain neurofilament antisera at 1:500 dilutions. Magnification $\times 640$.

triplet proteins but rather a protein in the microtubule fraction which migrates ahead of (i.e., is smaller than) tubulin. Eng et al. (1980) reported that antisera raised to gel-purified tubulin and to reassembled tubulin preparations containing microtubule-associated proteins did not stain Alzheimer's tangles preferentially.

In related studies, Grundke-Iqbal et al. (1979b) have reported the raising of an antiserum to a gel-purified, approximately 50,000 -dalton polypeptide present in paired helical filament-enriched fractions prepared from Alzheimer's cortex. This antiserum also was found to stain Alzheimer's neurofibrillary tangles, using the PAP method on paraffin-embedded sections. The antiserum did not stain astrocytes. The relationship of the 50,000dalton protein used to raise this antiserum and the protein within the microtubule fraction giving rise to tanglestaining activity has not yet been established (Iqbal et al., 1980).

The antisera used by Ishii et al. (1979) were raised in rabbits to the main 50,000-dalton component of calf brain filament preparations isolated according to Yen et al. (1976). The antisera so obtained were mixed; that is, they reacted with both neurons and glia in cryostat sections of human and rat brain. It has been shown by immunoaffinity chromatography that the major fraction of the 50,000 -dalton component in such brain filament preparations is GFA protein, the subunit of glial-specific filaments. However, a smaller fraction of the $\sim 50,000$-dalton component did not attach to anti-GFA immuno-Sepharose and thus was interpreted tentatively as a neurofila- ment degradation product (Dahl and Bignami, 1979). A direct demonstration of the existence of neurofilament degradation products in the 50,000-dalton range has been obtained recently by immunoaffinity chromatography with neurofilament antisera (Dahl, 1981). As to the production of mixed antisera (anti-GFA and antineurofilament) with the $\sim 50,000$-dalton fraction of brain filament protein (Ishii et al., 1979), similar results have been reported by Liem et al. (1978). It also appears that the antisera obtained by Eng and Rubinstein (1978) with a similar antigen (bovine spinal cord $\sim 50,000$-dalton protein) also were mixed. Figure 1 in Eng et al. (1980) illustrates staining of Purkinje cell baskets and of nerve fibers in the inner half of the molecular layer of rat cerebellum in addition to Bergmann glia. In our hands, rabbits injected with the $\sim 50,000$-dalton protein isolated by SDS-PAGE from bovine brain filament preparations have produced GFA antisera with no antineurofilament activity (Dahl, 1980b) and the same results have been reported by Yen and Fields (1981) with human antigen obtained by the same procedure. At the other end of the spectrum, mice injected with the $\sim 50,000$-dalton bovine filament protein have produced neurofilament antisera with no anti-glia activity (Schachner et al., 1978).

To summarize the issue, it appears that the $\sim 50,000$ dalton component in brain filament preparations is heterogeneous and that experimental animals injected with this antigen may produce antisera to neurofilament degradation products constituting a small fraction of the preparation. In our opinion, this is the explanation for 


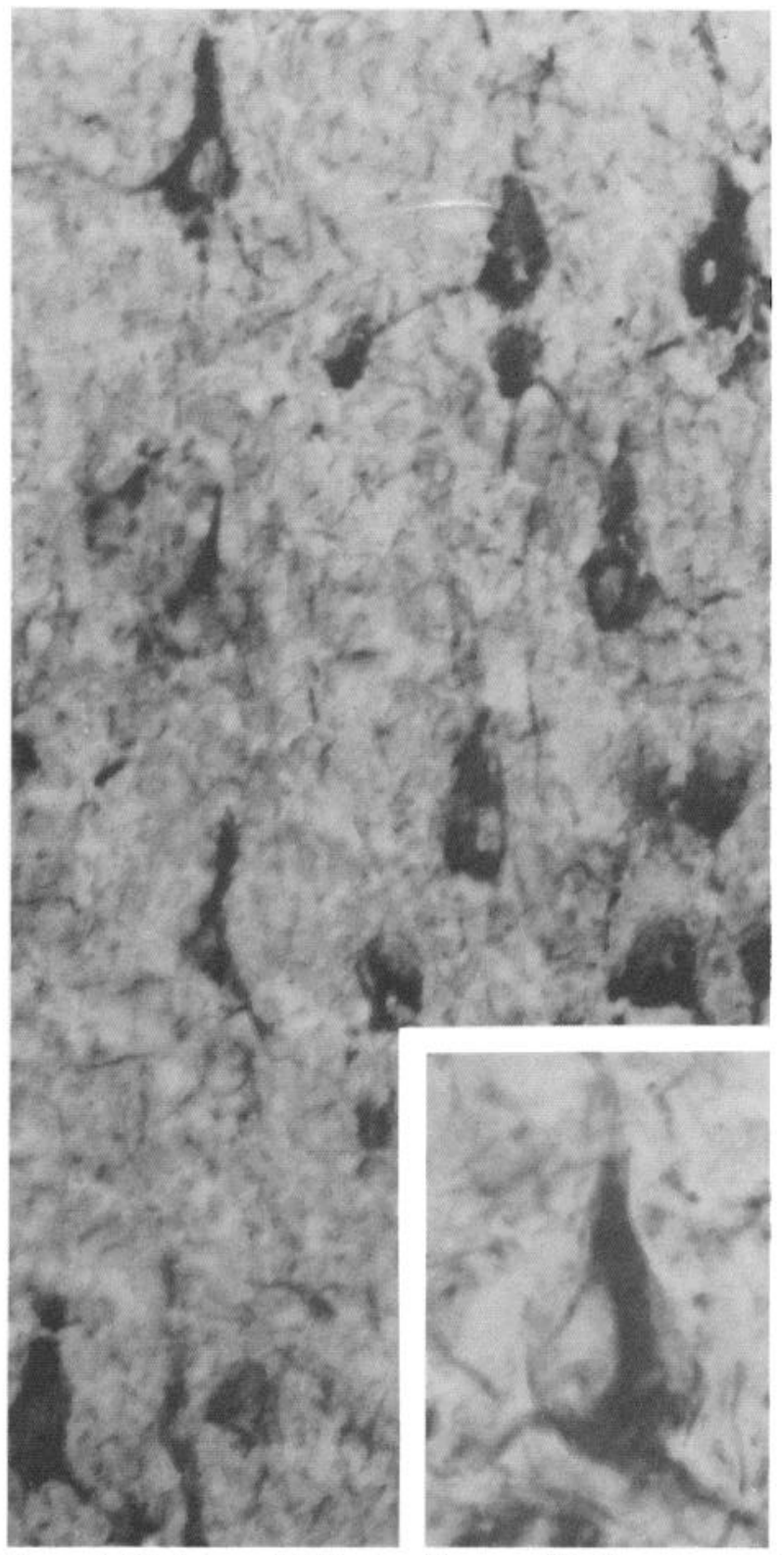

Figure 3. Staining of Alzheimer's neurofibrillary tangles in the pyramidal layer of the hippocampus using the PAP procedure with anti-chicken brain neurofilament antisera at 1:500 dilutions (same case as in Fig. 2). Magnification $\times 250$ and $\times$ 640 (inset).

the immunohistological staining of Alzheimer's neurofibrillary tangles reported by Ishii et al (1979).

The paper by Gambetti et al. (1980) is more relevant to the present communication since the staining of Alzheimer's neurofibrillary tangles was obtained with the same antisera as employed in this study, that is, antisera raised to antigens isolated by hydroxyapatite chromatography from $8 \mathrm{~m}$ urea extracts of chicken brain. We repeated the work for two reasons. First, neurofibrillary tangles were shown by Gambetti et al. (1980) to react with antiserum to the chicken brain preparation using the PAP procedure in formalin-fixed, paraffin-embedded material. In contrast, we have not been able to obtain neurofilament staining in paraffin-embedded tissues, not only with human autopsy material but also with formalin-perfused rats and rabbits. In our hands, neurofilament staining could be obtained only in cryostat sections and in Vibratome sections of formalin-perfused brain (Dahl et al., 1981a, b). Second, staining of neurofibrillary tangles in paraffin-embedded material could be obtained by Gambetti et al. (1980) only with antiserum from one of three immunized rabbits, all of which produced antisera that strongly stained normal neurofilaments. Using acetone-fixed cryostat sections, we have found that antisera from all three rabbits react with the tangles. Furthermore, we have not detected any difference among these three antisera by double immunodiffusion, immunoaffinity chromatography, and immunohistology on cryostat sections. Although the reasons for these discrepancies between our present studies and the earlier work of Gambetti et al. (1980) remain unclear, we believe that our findings strongly support the conclusion reached in the previous study that "the main component of Alzheimer neurofibrillary tangles appears to be a protein antigenically related to neurofilament protein" (Gambetti et al., 1980).

The data reviewed above lead to a discussion of the relationship between normal neurofilaments and the filaments forming Alzheimer's neurofibrillary tangles. It is presently believed that a triplet of polypeptides at approximately $200,000,150,000$, and 70,000 daltons constitute the mammalian neurofilament (see Lazarides, 1980, for review). The anti-chicken brain antisera which strongly stained neurofibrillary tangles reacted with the 70,000 - and 150,000-dalton neurofilament polypeptides by immunoaffinity chromatography (Dahl, 1981) and with all components of the neurofilament "triplet" $(70,000,150,000$, and 200,000$)$ by immunolabeling of gels. Conversely, Alzheimer's neurofibrillary tangles are stained weakly with an antiserum raised to the 150,000 dalton neurofilament protein isolated by SDS-acrylamide gel electrophoresis from brain filament preparations. Similar results with antisera to gel-purified neurofila-

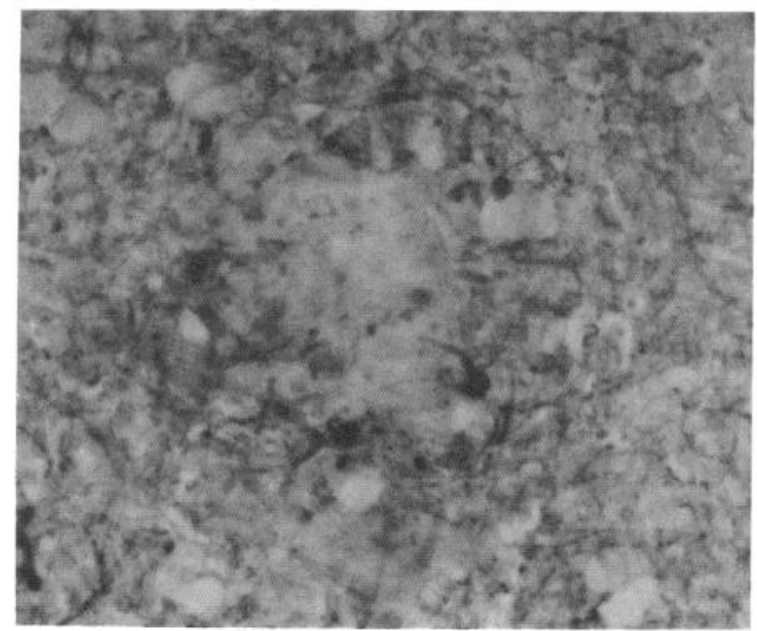

Figure 4. Staining of distended small nerve processes probably surrounding the central core of a senile plaque in the fascia dentata of hippocampus using the PAP procedure with antichicken brain neurofilament antisera at 1:500 dilutions. Magnification $\times 640$. 


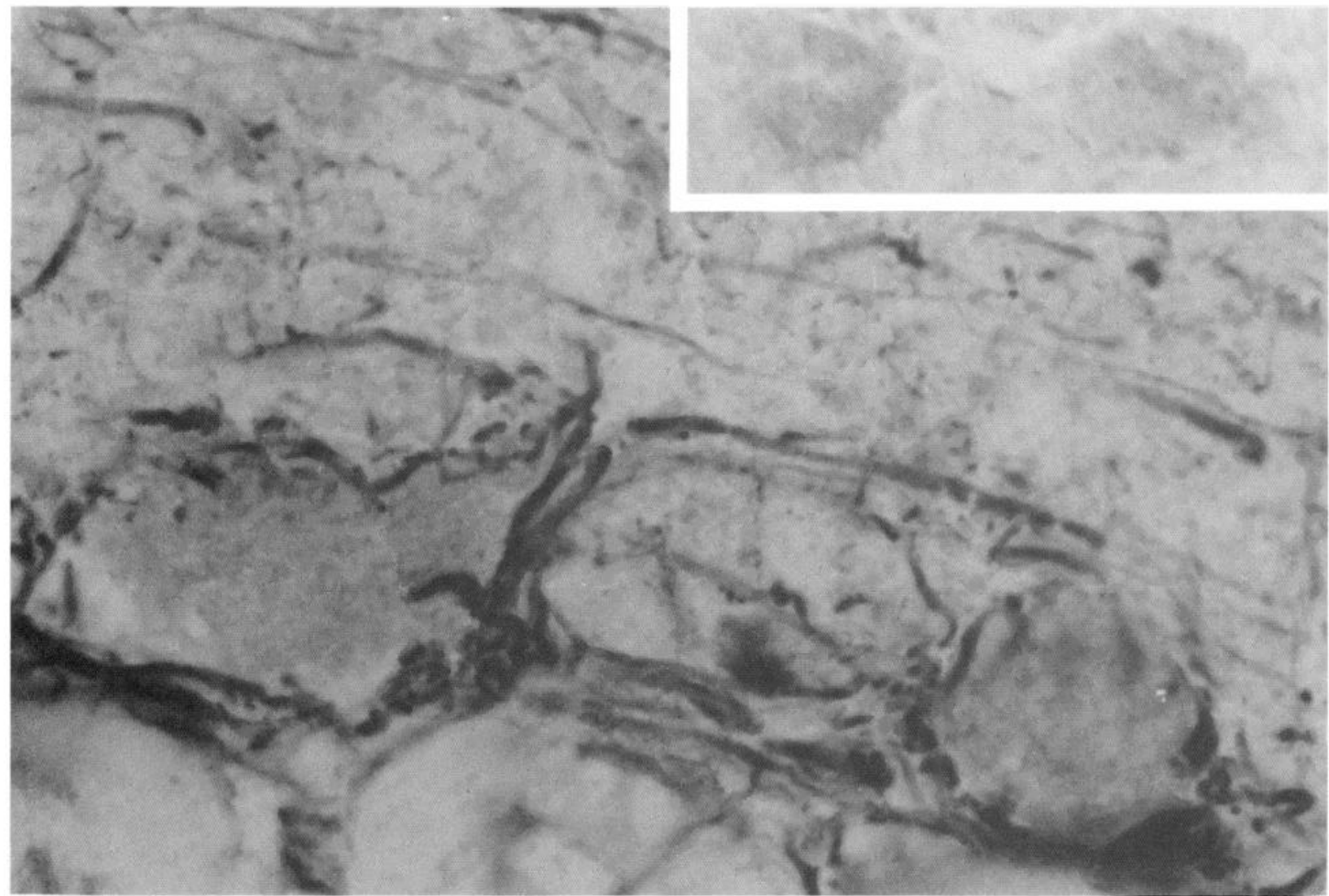

Figure 5. Staining of Purkinje cell baskets and of nerve fibers in the inner half of the molecular layer using the PAP procedure with antisera to bovine 150,000-dalton neurofilament protein at 1:100 dilutions. Inset, Weak staining of Alzheimer's neurofibrillary tangles under the same conditions. Magnification $\times 640$.

ment proteins have been reported previously (Gambetti et al., 1980).

Another point for discussion is the immunological relatedness of neurofibrillary tangles in Alzheimer's disease and in rabbits injected intrathecally with aluminum or mitotic spindle inhibitors (the latter appear to be formed of morphologically normal 10-nm filaments). According to Dahl et al. $(1980,1981 a)$, experimental neurofibrillary tangles displayed similar properties; i.e., they stained with anti-chicken brain neurofilament antisera and failed to react with antisera to gel-purified 150,000-dalton neurofilament protein. In contrast, Selkoe et al. (1979) did find bright immunofluorescent staining of aluminum-induced neurofibrillary tangles with antisera raised by the same procedure, i.e., antisera to gel-purified 150,000 -dalton neurofilament protein.

In conclusion, we believe that the immunological crossreactivity of Alzheimer's neurofibrillary tangles and normal neurofilament protein(s) is now firmly established. Structural alterations of neurofilaments, similar to those observed in Alzheimer's disease have been produced experimentally by prolonged incubation in $0.1 \mathrm{M} \mathrm{KCl}$ of neurofilament-enriched fractions isolated from rat peripheral nerve (Schlaepfer, 1978). It is interesting to note that, in 1907, Alzheimer considered that the change affected the neurofibrils present in the nerve cell (Corsellis, 1976) and that these neurofibrils correspond to neurofilaments at the electron microscopic level (Guillery, 1970).

\section{References}

Ball, M. J. (1976) Neurofibrillary tangles and the pathogenesis of dementia: A quantitative time study. Neuropathol. Appl. Neurobiol. 2: 394-410.

Corsellis, J. A. N. (1976) Aging and the dementias. In Greenfield's Neuropathology, W. Blackwood and J. A. N. Corsellis, eds., pp. 796-848, Edward Arnold, London.

Dahl, D. (1980a) Astroglial and axonal proteins in isolated brain filaments. II. Isolation of a 70,000 dalton polypeptide from brain filament preparations of bovine brain by immunoaffinity chromatography with antineurofilament antisera. Biochim. Biophys. Acta 622: 9-17.

Dahl, D. (1980b) Study on the immunological crossreactivity of neurofilament polypeptides in axonal preparations of bovine brain. FEBS Lett. 111: 152-156.

Dahl, D. (1981) Isolation of neurofilament proteins and of immunologically active neurofilament degradation products from extracts of brain, spinal cord and sciatic nerve. Biochim. Biophys. Acta 668: 299-306.

Dahl, D., and A. Bignami (1976) Immunogenic properties of the glial fibrillary acidic protein. Brain Res. 116: 150-157.

Dahl, D., and A. Bignami (1977) Preparation of antisera to neurofilament protein from chicken brain and human sciatic nerve. J. Comp. Neurol. 176: 645-658.

Dahl, D., and A. Bignami (1979) Astroglial and axonal proteins in isolated brain filaments. I. Isolation of the glial fibrillary acidic protein and of an immunologically active cyanogen bromide peptide from brain filament preparations of bovine white matter. Biochim. Biophys. Acta 578: 305-316.

Dahl, D., and A. Bignami (1981) Immunohistological localiza- 
tion of desmin, the muscle-type $100 \AA$ filament protein, in rat astrocytes and Müller glia. J. Histochem. Cytochem., in press.

Dahl, D., A. Bignami, N. T. Bich, and N. H. Chi (1980) Immunohistochemical characterization of neurofibrillary tangles induced by mitotic spindle inhibitors. Acta Neuropathol. (Berl.) 51: 165-168.

Dahl, D., A. Bignami, N. T. Bich, and N. H. Chi (1981a) Immunohistochemical localization of the $150 \mathrm{~K}$ neurofilament protein in the rat and the rabbit. J. Comp. Neurol. 195: 659666.

Dahl, D., B. T. Nguyen, and A. Bignami (1981b) Ultrastructural localization of neurofilament proteins in aluminum-induced neurofibrillary tangles and rat cerebellum by immunoperoxidase labeling. Dev. Neurosci., in press.

De Vries, G. H., W. T. Norton, and C. S. Raine (1972) Axons: Isolation from mammalian central nervous system. Science 175: 1370-1372.

Eng, L. F., and L. J. Rubinstein (1978) Contribution of immunohistochemistry to diagnostic problems of human cerebral tumors. J. Histochem. Cytochem. 26: 513-522.

Eng, L. F., L. S. Forno, J. W. Bigbee, and K. I. Forno (1980) Immunohistochemical localization of glial fibrillary acidic protein and tubulin in Alzheimer's disease brain biopsy material. In Aging of the Brain and Dementia, L. Amaducci, A. N. Davison, and P. Antuono, eds., pp. 49-54, Raven Press, New York.

Farmer, P. M., A. Peck, and R. D. Terry (1976) Correlations among numbers of neuritic plaques, neurofibrillary tangles, and the severity of senile dementia. J. Neuropathol. Exp. Neurol. 35: 367.

Franke, W. W., E. Schmid, M. Osborn, and K. Weber (1978) Different intermediate-sized filaments distinguished by immunofluorescence microscopy. Proc. Natl. Acad. Sci. U.S. A. 75: 5034-5038.

Gambetti, P., M. E. Velasco, D. Dahl, A. Bignami, U. Roessmann, and S. D. Sindely (1980) Alzheimer neurofibrillary tangles: An immunohistochemical study. In Aging of the Brain and Dementia, L. Amaducci, A. N. Davison, and P. Antuono, eds., pp. 55-63, Raven Press, New York.

Gröschel-Stewart, U., F. J. Schreiber, C. Mahlmeister, and K. Weber (1976) Production of specific antibodies to contractile proteins and their use in immunofluorescence microscopy. I. Antibodies to smooth and striated chicken muscle myosins. Histochemistry 46: 229-239.

Grundke-Iqbal, J., A. Johnson, H. M. Wisniewski, R. D. Terry, and K. Iqbal (1979a) Evidence that Alzheimer neurofibrillary tangles originate from neurotubules. Lancet 1: 578-579.

Grundke-Iqbal, J., A. B. Johnston, R. D. Terry, H. M. Wisniewski, and K. Iqbal (1979b) Alzheimer neurofibrillary tangles: Antiserum and immunohistological staining. Ann. Neurol. 6: $532-537$.

Guillery, R. W. (1970) Light- and electron-microscopical studies of normal and degenerating axons. In Contemporary $R e$ search Methods in Neuroanatomy, W. J. H. Nauta and S. D. E. Ebbesson, eds., pp. 77-105, Springer-Verlag, New York.

Hoffman, P. N., and R. L. Lasek (1975) The slow component of axonal transport. Identification of major structural polypeptides of the axon and their generality among mammalian neurons. J. Cell Biol. 66: 351-366.

Iqbal, K., J. Grundke-Iqbal, A. B. Johnson, and H. M. Wisniewski (1980) Neurofibrous proteins in aging and dementia. In Aging of the Brain and Dementia, L. Amaducci, A. N.
Davison, and P. Anatuono, eds., pp. 39-48, Raven Press, New York.

Ishii, T., S. Haga, and S. Tokutake (1979) Presence of neurofilament protein in Alzheimer's neurofibrillary tangles (ANT). An immunofluorescent study. Acta Neuropathol. (Berl.) 48: 105-112.

Katzman, R. (1976) The prevalence and malignancy of Alzheimer disease. Arch. Neurol. 33: 217-218.

Kidd, M. (1963) Paired helical filaments in electron microscopy of Alzheimer's disease. Nature 197: 192-193.

Lazarides, E. (1980) Intermediate filaments as mechanical integrators of cellular space. Nature 283: 249-256.

Liem, R. K. H., S. -H. Yen, G. D. Salomon, and M. L. Shelanski (1978) Intermediate filaments in nervous tissue. J. Cell Biol. 79: 637-645.

Schachner, M., C. Smith, and G. Schoonmaker (1978) Immunological distinction between neurofilament and glial fibrillary acidic proteins by mouse antisera and their immunohistological characterization. Dev. Neurosci. 1: 1-14.

Schlaepfer, W. W. (1978) Deformation of isolated neurofilaments and pathogenesis of neurofibrillary pathology. J. Neuropathol. Exp. Neurol. 37: 244-254.

Schlaepfer, W. W., and L. A. Freeman (1978) Neurofilament proteins of rat peripheral nerve and spinal cord. J. Cell Biol. 78: 653-662.

Selkoe, D. J., R. K. H. Liem, S. -H. Yen, and M. L. Shelanski (1979) Biochemical and immunological characterization of neurofilaments in experimental neurofibrillary degeneration induced by aluminum. Brain Res. 163: 235-252.

Terry, R. D. (1963) The fine structure of neurofibrillary tangles in Alzheimer's disease. J. Neuropathol. Exp. Neurol. 22: 629642 .

Thorpe, R., A. Delacourte, and B. H. Anderton (1979) The isolation of brain $10 \mathrm{~nm}$ filament polypeptides from ureaextracts of brain white matter. FEBS Lett. 103: 148-151.

Tomlinson, B. F., G. Blessed, and M. Roth (1970) Observation of the brains of demented old people. J. Neurol. Sci. 11: 205243.

Van Raamsdonk, W., C. W. Pool, and C. Heytinu (1977) Detection of antigens and antibodies by an immunoperoxidase method applied on thin longitudinal sections of SDS-polyacrylamide gels. J. Immunol. Methods 17: 337-348.

Weber, K., and M. Osborn (1969) The reliability of molecular weight determinations by dodecyl sulfate-polyacrylamide gel electrophoresis. J. Biol. Chem. 244: 4406-4412.

Wiśniewski, H. M., and R. D. Terry (1973) Reexamination of the pathogenesis of the senile plaque. Prog. Neuropathol. 2: $1-26$.

Wiśniewski, H. M., B. Ghetti, and R. D. Terry (1973) Neuritic (senile) plaques and filamentous changes in aged rhesus monkeys. J. Neuropathol. Exp. Neurol. 32: 566-584.

Wiśniewski, H. M., H. K. Narang, and R. D. Terry (1976) Neurofibrillary tangles of paired helical filaments. J. Neurol. Sci. 27: 173-181.

Yen, S. -H., and K. L. Fields (1981) Antibodies to neurofilament, glial filament and fibroblast intermediate filament proteins bind to different cell types of the nervous system. J. Cell Biol. 88: 115-126.

Yen, S. -H., D. Dahl, M. Schachner, and M. L. Shelanski (1976) Biochemistry of the filaments of brain. Proc. Natl. Acad. Sci. U. S. A. 73: 529-533. 